\title{
Kann man schlechte Laune einfach
} wegessen?

Wie Omega-3-Fettsäuren und Vitamin D die Stimmung beeinflussen

Volker Schmiedel

\section{Depression immer mehr im Fokus}

Erinnern Sie sich noch an Robert Enke? Ein erfolgreicher und beliebter Sportler auf dem Wege zum Nationalstammtorwart, von Presse, Fans, Trainern und Mitspielern geachtet, ohne Skandale oder private Probleme - und er bringt sich ohne erkennbaren Anlass um. Vor Kurzem erschütterte die Nachricht vom Suizid des Schauspielers Robin Williams nicht nur die Filmwelt - ein Oscar-Preisträger, der in vielen Filmen zahllose Zuschauer zum Lachen brachte, beendet freiwillig sein Leben. Und ganz aktuell: Ein Flugzeug zerschellt in den Alpen. Die Untersuchungen ergeben schnell, dass Andreas Lubitz, einer der Piloten, willentlich den Absturz herbeiführte und alle Insassen mit in den Tod riss.

Sind das tragische Einzelfälle oder nur die auffällige Spitze des Eisbergs „Volkskrankheit Depression“? Die epidemiologischen Daten stützen leider letztere Vermutung:

- Jeder 2. Mensch in Deutschland hat irgendwann einmal im Laufe seines Lebens mindestens eine depressive Episode.

- Jeder 10. leidet jährlich mindestens an einer depressiven Episode.

- Jede 5. depressive Episode geht in eine chronische Depression über.

- Jeder 3. derjenigen, die nicht in die chronische Phase übergehen, erleidet innerhalb von 2 Jahren einen Rückfall.

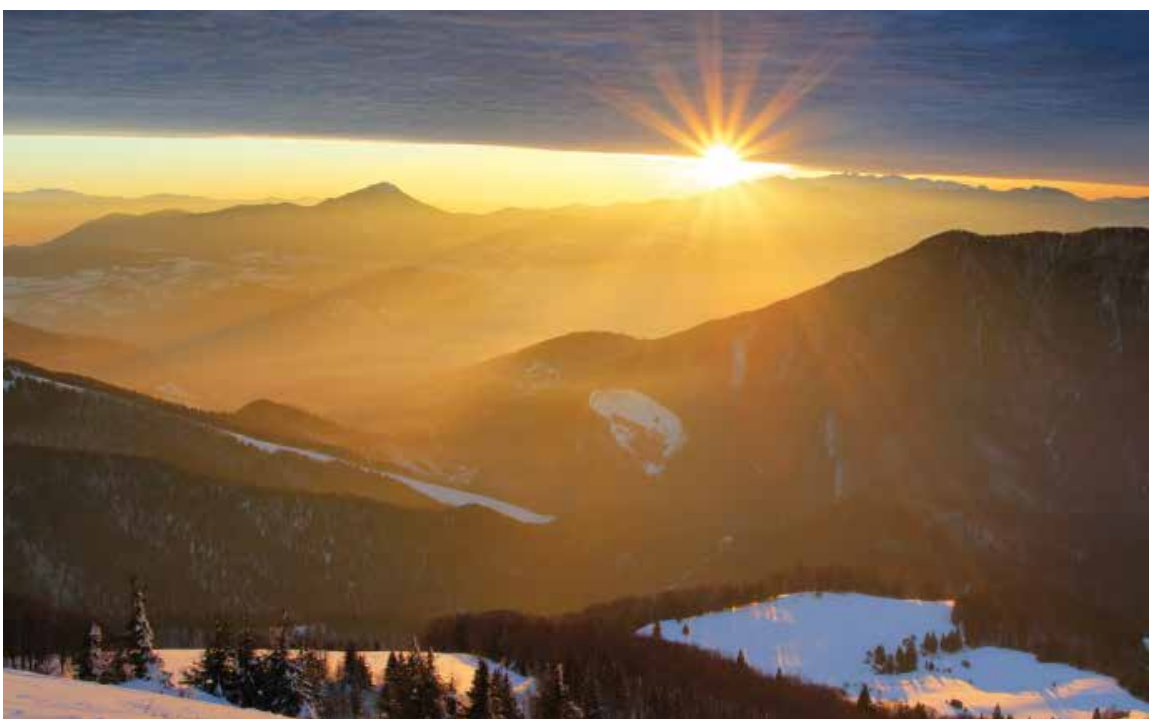

Abb. 1 Sonnenvitamin D - Das Hormon wirkt antiinflammatorisch und kann bei guter Versorgung das Depressionsrisiko senken. @ TTstudio/Fotolia

Während viele andere chronischen Erkrankungen auf dem Rückzug sind, steigen die Erkrankungszahlen bei Depressionen kontinuierlich an (Abb. 2).

\section{Helfen Fische gegen Depressionen?}

In einer Übersichtsarbeit geben Grosso et al. [3] eindeutige inverse Korrelation zwischen Fischkonsum und Depressivität an. Dasselbe gilt übrigens auch für Ängstlichkeit. Es muss also nicht heißen: „Keine Angst vor Fischen“, sondern „Keine Angst durch Fische“.
Eine interventionelle Studie [1] teilte 42 Patienten mit der Diagnose Major Depression kontrolliert, randomisiert und doppelblind in 2 Gruppen ein. Teilnehmer der einen erhielten $900 \mathrm{mg}$ EPA/300 mg DHA, die der anderen ein Placebopräparat. Beide erhielten mit Citalopram eine suffiziente, medikamentöse, antidepressive Therapie. Obwohl es bei einer potenten und wirksamen Intervention nicht ganz einfach ist, die Überlegenheit einer zusätzlichen Maßnahme statistisch nachzuweisen, zeigte sich bereits nach 4 Wochen bei der Verum-Gruppe ein signifikant besserer Depressionsscore (Hamilton-Score) auf $(\mathrm{p}=0,014)$, nach 8 Wochen war der Unterschied hochsignifikant $(\mathrm{p}<0,001)$. 
Neuere Forschungen weisen darauf hin, dass Depressionen - nicht nur, aber auch - Entzündungskrankheiten sind. Zwei wichtige antiinflammatorische Substanzen sind Omega-3-Fettsäuren und das Vitamin D. Nach epidemiologischen Studien ist das Risiko für eine Depression bei guter Versorgung geringer. Interventionell können schnellere Therapieerfolge bei optimaler Versorgung mit diesen Nährstoffen erzielt werden. In diesem kurzen Review werden einige Studien sowie eine Kasuistik vorgestellt.

Schlüsselwörter

Depression, Omega-3-Fettsäuren, Vita$\min \mathrm{D}$.

Abb. 3 zeigt, dass die Patienten, die Fischöl erhielten, bereits nach 4 Wochen die Depressionsscores erreichten, die den Werten der Placebogruppe (korrekt: die Gruppe mit Citalopram allein) nach 8 Wochen entsprachen. Man kann also durchaus das Eine tun, ohne das andere zu lassen.

Eine hohe Zufuhr an Omega-3-Fettsäuren maritimer Herkunft scheint die Wirkung konventioneller Antidepressiva zu verstärken. Eine durchaus nebenwirkungsbehaftete Therapie mit Antidepressiva vielleicht 4 Wochen früher beenden zu können, stellt für betroffene Patienten eine attraktive Option dar.

\section{ABSTRACT}

Recent research suggests that depression is an inflammatory disease - not only, but also. Two important anti-inflammatory substances are omega-3 fatty acids and vitamin D. According to epidemiological studies, the risk of depression in good supply is low. Therapeutic results can be achieved faster with an optimum supply of these nutrients. In this brief review some studies and a case report will be presented.

\section{Keywords}

Depression, omega-3-fatty acids, vitamin D.

Hilft Omega-3 vielleicht auch ohne Antidepressiva? Bei einer potenziell lebensgefährlichen Erkrankung wie einer schweren Depression ist es ethisch nicht vertretbar, leidende Patienten wochenlang mit einer (noch nicht bewiesenen) Therapie oder mit (gar nichts) Placebo zu behandeln. Folgende Studie hat trotzdem die Wirksamkeit einer alleinigen Omega-3Therapie in einem ethisch vertretbaren Design bewiesen. Krawczyk et al. [5] behandelten 21 Patienten mit schwerer Depression, die auf eine jeweils mindestens 4-wöchige Therapie mit $300 \mathrm{mg}$ Venlafaxin und anschließend $60 \mathrm{mg}$ Paroxetin nicht angesprochen hatten, im pharmakologischen Sinne also als therapieresistent

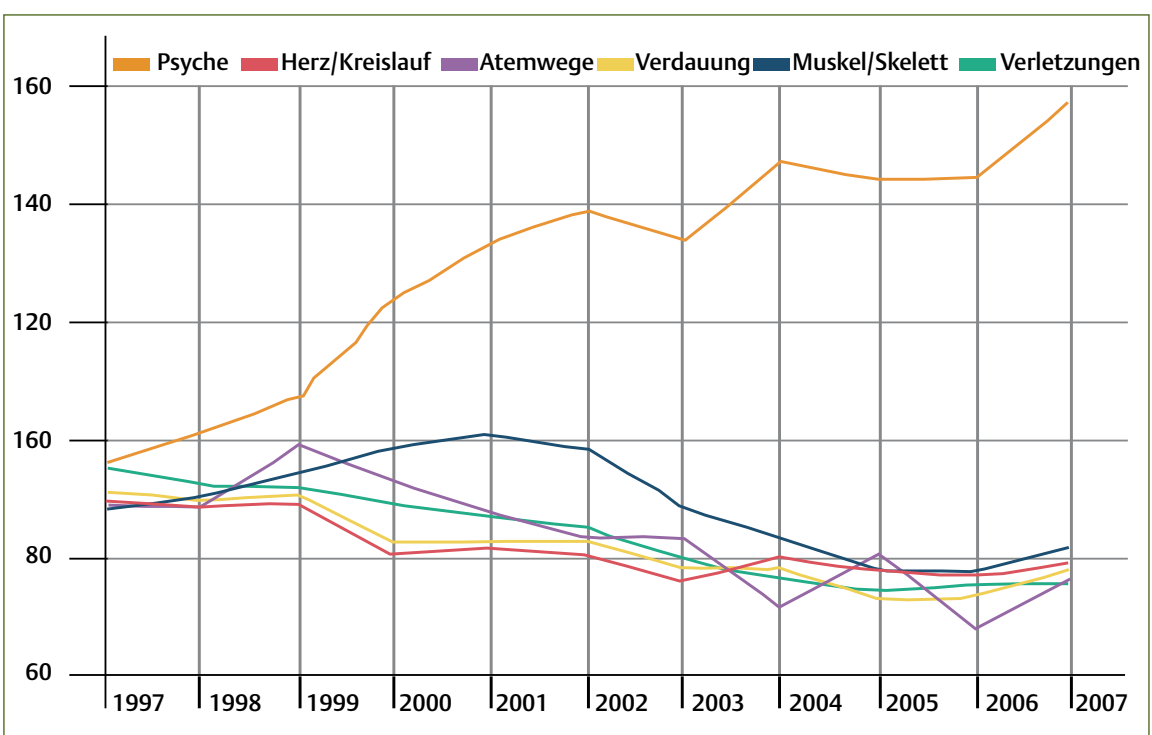

Abb. 2 Zu- bzw. Abnahme einiger wichtiger Krankheiten. @ c www.psychenet.de galten, mit Omega-3-Fettsäuren. Die dabei verwendete Dosis lag mit $3 \mathrm{~g}$ Omega-3 (=24 Fischölkapseln) in einem wirklich hohen Bereich. Es wurde leider nichts darüber berichtet, ob und wie die depressiven Patienten diese große Menge toleriert haben.

\begin{abstract}
Tipp
Ich persönlich gebe bei Erkrankungen mit einem nachgewiesenen oder wahrscheinlichen Effekt von Omega-3 wie etwa Herzrhythmusstörungen, Rheuma oder eben auch Depressionen komplementär zu konventioneller Therapie ein qualitativ hochwertiges Fischöl mit einem reinen Omega-3-Anteil von ca. $2 \mathrm{~g}$ pro EL. In der oben erwähnten Studie wäre man also leicht mit $1 \frac{1}{2} \mathrm{EL}$ ausgekommen - eine Dosis, die in Smoothie, Suppe oder Soße von Patienten problemlos verzehrt werden kann. Mit einer vorherigen Fettsäureanalyse kann man die benötigte Menge noch genauer abschätzen.
\end{abstract}

Zurück zur Studie: Die zuvor therapieresistenten Patienten mit Depressionen erzielten innerhalb von nur 4 Wochen eine Normalisierung ihrer Depressionswerte. Der HRDS ging in dieser kurzen Zeit (nach 8-wöchiger frustraner Therapie mit zwei verschiedener synthetischen Antidepressiva in ansonsten suffizienter Dosierung!) von 30 auf 11 zurück. Auch wenn hier eine Kontrollgruppe fehlt, dürfen aufgrund des intraindividuellen historischen Vergleichs Placeboeffekte als alleinige Erklärung für diese dramatische Besserung kaum herangezogen werden können.

\section{Hilft das Sonnenvitamin D auch bei Depression?}

Seit Längerem ist bekannt, dass eine Beziehung zwischen Depression und Arteriosklerose besteht. In dieser Studie untersuchten May et al. [6] 7358 Patienten, die 50 Jahre oder älter waren und bei denen eine entsprechende Diagnose (z.B. KHK), aber anamnestisch keine bekannte Depression vorlag. Der gemessene Vitamin-D-Spiegel wurde in 4 Kategorien ein- 
geteilt: $>125 \mathrm{nmol} / \mathrm{l}=0$ (optimal), $>75-$ $125 \mathrm{nmol} / \mathrm{l}=\mathrm{N}$ (normal), $>37,5-75 \mathrm{nmol} / 1$ $=\mathrm{L}$ (low, niedrig), $\leq 37,5 \mathrm{nmol} / \mathrm{l}=\mathrm{VL}$ (very low, sehr niedrig). Das Risiko, depressiv zu werden, wurde in der Gruppe mit optimalen Werten gleich 1 gesetzt und das relative Risiko in den anderen Gruppen berechnet, wobei sich folgende Werte ergaben (Abb. 4).

Je niedriger der Vitamin-D-Spiegel lag, umso höher war das Risiko, eine neue Depression zu entwickeln. Zwischen der Gruppe mit optimalen und sehr niedrigen Werten betrug der Unterschied das 2,7-fache $(p=0,005)$. Erstaunlich ist bei dieser Untersuchung, dass es noch einen deutlichen Unterschied zwischen normalen und optimalen Werten gab, wobei die normalen Werte mit 75-125 nmol/l sogar im hochnormalen Bereich lagen. In der „normalen“ Gruppe entwickelten sich immerhin fast doppelt so viele Depressionen wie in der „optimalen“ Gruppe, was ein Hinweis darauf ist, dass möglicherweise höhere Werte (über 100, nach dieser Studie sogar $125 \mathrm{mmol}$ ) angestrebt werden sollten.

\section{Tipp}

Bei allen „Vitamin-D-abhängigen Erkrankungen" strebe ich einen Vitamin-DSpiegel von 40-60 ng/ml bzw. 100$150 \mathrm{nmol} / \mathrm{l}$ an. Aufgrund dieser Untersuchung versuche ich, depressive Patienten auf einen hochnormalen Spiegel an die obere Normgrenze oder sogar knapp darüber zu bringen. Hierfür sind bei den meisten Patienten allerdings Dosen von ca. 3000-6000 IE pro Tag erforderlich.

Von der beginnenden „toxischen Laborgrenze“ von $100 \mathrm{ng} / \mathrm{ml}$ bzw. $250 \mathrm{nmol} / \mathrm{l}$ sind wir damit immer noch weit entfernt. Sicherheitshalber sollten wir aber bei von vielen Apothekern und Ärzten immer noch als „Mega-Dosen“ betrachteten Mengen von über 2000 IE nach 2-3 Monaten eine Serumkontrolle des Vitamin-D-Spiegels veranlassen, um forensisch nicht angreifbar zu sein und die Dosis noch feinjustieren zu können, da die Ansprechrate auf Vitamin D eine große individuelle Streubreite aufweist.

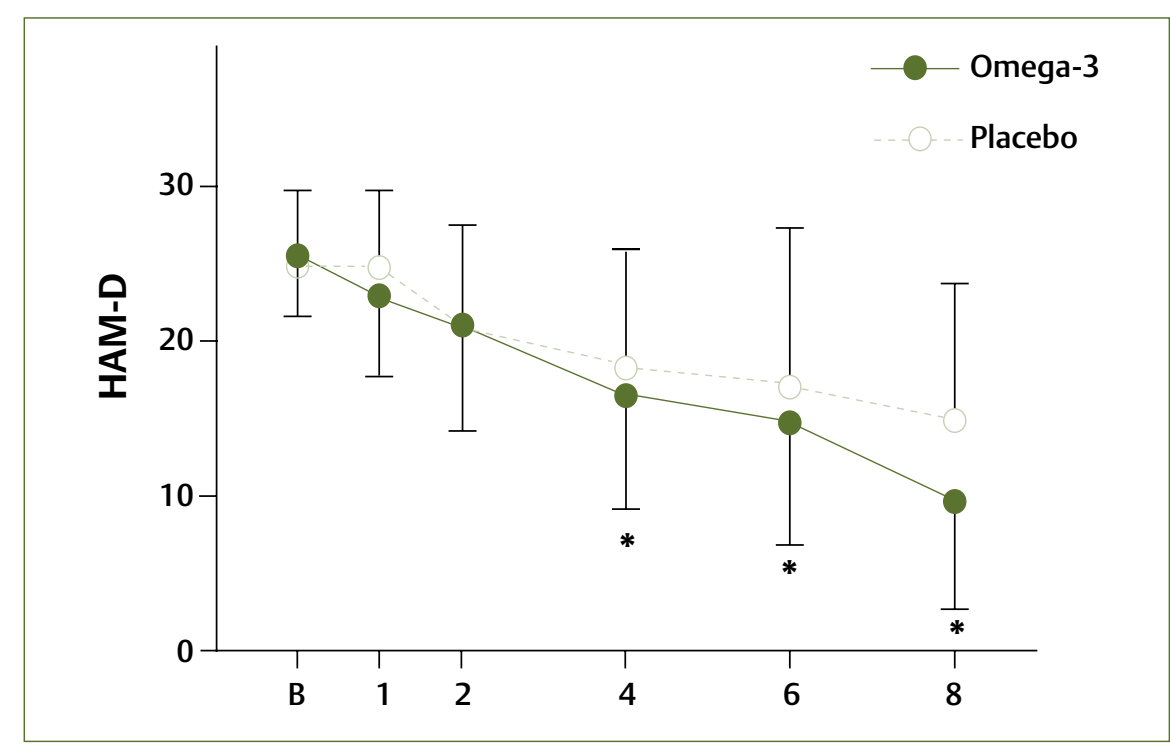

Abb. 3 Rückgang des Hamilton-Depression-Scores (HRDS) unter Citalopram mit oder ohne Omega-3.

In einer Interventionsstudie fanden Jorde et al. [4] bei immerhin 441 depressiven Erwachsenen zwischen 21 und 70 Jahren noch Unterschiede im Ausmaß der Depressivität in Abhängigkeit vom Vitamin-D-Spiegel. Lag der Vitamin-D-Spiegel unter $75 \mathrm{nmol} / \mathrm{l}$ wurde ein Beck-Depression-Score von 6,0 gefunden, bei einem Spiegel über $75 \mathrm{nmol} / \mathrm{l}$ lag er nur bei 4,0.

Offensichtlich wird bei einer guten Versorgung nicht nur die Wahrscheinlichkeit einer Depression gesenkt, tritt dennoch eine Depression auf, ist diese immerhin nicht so schwer. Die Patienten erhielten anschließend ein Jahr lang 20000 oder 40000 IE Vitamin pro Woche oder Placebo. Eine signifikante Verbesserung des BeckDepression-Score wurde nur in den Vitamin-D-Gruppen beobachtet.

\section{Kasuistik: 51-jährige Frau mit Depression}

Ute G. ist eine inzwischen 51-jährige Patientin, die ich seit mehreren Jahren wegen Depression begleitend zu ihrer konventionellen Therapie (Medikamente, Psychotherapie) behandle. Bei der Erstuntersuchung vor etwa 5 Jahren nahm sie Citalopram $10 \mathrm{mg}$ ein, war mit dieser Therapie aber nicht zufrieden, da sie sich immer noch depressiv fühlte, andererseits aber wegen Nebenwirkungen der antide- pressiven Therapie die Dosis nicht noch steigern, sondern nach Möglichkeit eher reduzieren wollte.

\section{Orthomolekulare Therapie: \\ Mineralstoffe, Vitamine}

In der ausführlichen Nährstoffdiagnostik fand sich ein Mangel an Kalium, Magnesium (wegen leichter Hypertonie, im Vollblut gemessen), Vitamin $\mathbf{B}_{6}$, Zink (alle im Vollblut gemessen) sowie Vitamin D (Serum). Sie wurde mit $300 \mathrm{mg}$ Magnesium, $1000 \mathrm{mg}$ Kalium und $20 \mathrm{mg}$ Zink (alles täglich) und $100 \mathrm{mg}$ Vitamin $\mathrm{B}_{6}$ sowie 20000 IE Vitamin D (beides wöchentlich) substituiert. Hierunter gab sie eine deutliche Verbesserung ihrer Beschwerden an und reduzierte selbst das Citalopram auf nur noch jeden zweiten Tag. Ich halte meine Patienten immer dazu an, eine Dosisreduktion oder gar ein Absetzen der antidepressiven Medikation immer mit dem behandelnden Psychotherapeuten oder Psychiater zu besprechen.

\section{Orthomolekulare Therapie: \\ Aminosäure}

Nach 2 Jahren ging es der Patientin subjektiv so gut, dass sie einen Auslassversuch wagen wollte. Gleichzeitig gab ich ihr $2 \mathrm{~g}$ Tryptophan, da sie klinische Hinweise auf einen Serotoninmangel aufwies. Typische Symptome eines Serotoninmangels sind Depressivität, Durchschlafstörungen, 


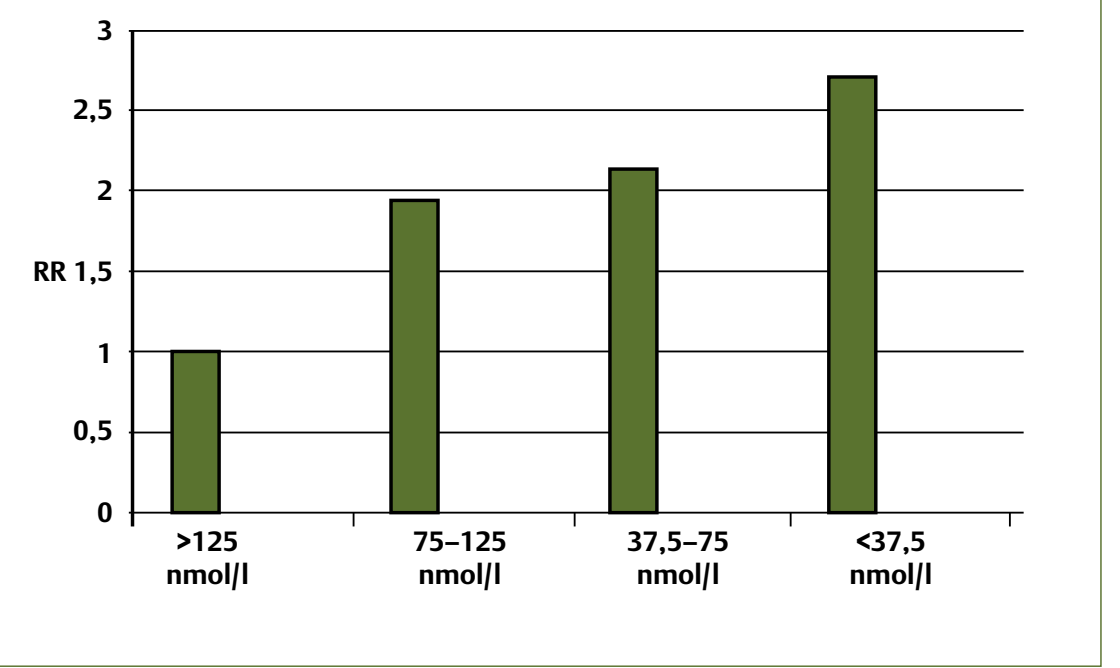

Abb. 4 Relatives Risiko für eine Depression in Abhängigkeit vom Vitamin-D-Spiegel.

Ängstlichkeit, Schmerzempfindlichkeit und geradezu als wegweisendes Symptom ein überdurchschnittliches Verlangen nach Süßem.

Wenn keine Tafel Schokolade vor einem Patienten sicher ist, dann sollte zumindest ein Serotoninmangel in Erwägung gezogen werden.

Mit der Labormessung von Serotonin bin ich nicht immer glücklich, da ich mitunter normales Serotonin gemessen habe, Patienten aber eine typische Symptomatik aufwiesen und auf eine entsprechende orthomolekulare Therapie auch gut ange- sprochen haben. Die Klinik halte ich beim Serotonin daher für wichtiger als die Diagnostik!

Eine orthomolekulare Therapie des Serotoninmangels besteht aus den Serotonin-Präkursoren L-Tryptophan 1-2g oder 5HTP 100-200 mg (bei Schlafstörungen die Tagesdosis zur Nacht geben, ansonsten ggf. über den Tag verteilen), Vitamin $B_{6}$ und Zink, die als Co-Enzyme der Serotoninsynthese gelten, bei niedrigem Ferritin auch Eisen sowie die ebenfalls serotoninergen Omega-3-Fettsäuren. Da bei der Kombination mit den Serotoninstoffwechsel beeinflussenden Psychopharmaka wie Trizyklika oder SSRI mit L-Tryptophan oder 5HTP die Ausbildung eines Serotoninsyndroms möglich ist, sollte eine solche vermieden werden. Bei sehr niedrigen Dosen beider
Mittel und sehr engmaschiger Kontrolle des Patienten können erfahrene und verantwortungsvolle Ärzte nach entsprechender Aufklärung und Einwilligung des Patienten eine Kombination mit überlappendem Ausschleichen der Psychopharmaka wagen.

\section{Orthomolekulare Therapie: \\ Fettsäuren}

Frau G. berichtete auch nach Absetzen weiter gute Stimmung und Schlaf, fühlte sich aber unruhiger. Aus Kostengründen (Patientin ist gesetzlich versichert und wünscht die Kosten daher möglichst niedrig zu halten) war bisher auf eine Fettsäureanalytik und -therapie verzichtet worden. Da Ome-

ga-3-Fettsäuren eine in Herzratenvariabilitätsuntersuchungen nachgewiesene parasympathomimetische Wirkung haben [7] und ich empirisch bei meinen Patienten mehr Ruhe und Gelassenheit unter den mit Omega-3-Therapierten erlebe, habe ich ihr auch ohne Messung die Gabe von knapp 1 EL Fischöl empfohlen (sie wog deutlich weniger als $75 \mathrm{~kg}$ ).

Beim nächsten Termin nach einigen Monaten gab Frau G. eine subjektive Besserung an, die sie aber noch nicht als ideal empfand. Nun bestand ich auf einer Fettsäureanalyse, die einen im Vergleich zur Normalbevölkerung (unter einer guten Portion Fisch pro Woche AA/EPA-Quotient meist bei ca. 10) überdurchschnittlich guten $(\mathrm{AA} / \mathrm{EPA}=5,95)$, aber noch nicht idealen (bei Gesunden unter 5, bei Kranken unter 3, noch besser 2,5) Quotienten ergab. Es wurde ihr empfohlen, die Dosis doch auf mindestens 1 EL zu steigern und zusätzlich nach Möglichkeit mehr fette 
Kaltwasserfische zu verzehren. Nebenbefundlich war ein suboptimaler Ferritinwert von $19 \mathrm{ng} / \mathrm{ml}$ (Patientin neigt zu Menorrhagie) gefunden worden, der mit $14 \mathrm{mg}$ Eisen täglich behandelt wurde.

\section{Abschlusskontrolle}

Bei der Abschlusskontrolle im Sommer 2015 wurden alle vorher im Mangel befindlichen Vitalstoffwerte im Idealbereich gemessen (AA/EPA mit 3,29 nahezu optimal). Die Patientin fühlte sich subjektiv seit Monaten so gut wie seit 10 Jahren (trotz jahrelanger Citalopramtherapie) nicht mehr. Sie konnte wieder eine Beziehung zu einem Mann eingehen. Die Heirat sollte wenige Wochen nach unserem letzten Termin stattfinden. Frau G. behauptet, dass sie nicht glaube, ohne dieses subtile Nährstoffprogramm diese psychische Stabilität und den Aufbau einer neuen Beziehung erreicht zu haben.

\section{Fazit: Nährstoffe können bei Depression helfen}

Mit einer oben beschriebenen Nährstofftherapie können wir möglicherweise viele Depressionen vermeiden, in Kombination mit Psychopharmaka eine schnellere Remission erreichen und helfen, psychische Stabilität zu erhalten. Bei schweren Depressionen sollten aber auch die Grenzen der Möglichkeiten der orthomolekularen Therapie respektiert werden. Aus Rücksichtnahme auf die persönliche Sicherheit des Patienten (und die forensische Sicherheit des Arztes) sollten die Nährstoffe zunächst nur komplementär zur anerkannten konventionellen Therapie mit Psychopharmaka und/oder nicht-medikamentösen Therapien verabreicht werden. Bei sehr gutem Ansprechen kann bei engmaschiger Kontrolle und guter Führung des Patienten, der glaubhaft zusichert, Verschlechterungen zeitnah rückzumelden, und in Kooperation mit dem behandelnden Psychotherapeuten respektive Psychiater, kann auch eine Reduktion bzw. ein Ausschleichen der antidepressiven Medikation erwogen wer- den. Dann wäre aus der komplementären eine alternative Medizin geworden.

Insbesondere bei risikoreichen Interventionen - und Depressionen sind per se mit erhöhten Risiken behaftet, die nicht immer erkennbar sind (siehe die Fälle Enke, Robbins und Lubitz) - sollten die weisen Empfehlungen unserer antiken Altvorderen strenge Beachtung finden:

- Salus aegroti suprema lex. (Das Wohl des Kranken ist das oberste Gesetz.)

- Primum nil nocere. (Als erstes gilt: Nicht zu schaden.)

Wir dürfen aber auch unter Beachtung obigen Kautelen ein wenig mutig sein: Sapere aude. (Wage es, weise zu sein oder: Wage es, selbständig zu denken.)

In diesem Sinn: Viel Erfolg bei der Behandlung von Depressionen (auch) mit Nährstoffen.

Online zu finden unter:

http://dx.doi.org//10.1055/s-0041-109259

Interessenkonflikt: Der Autor hat in den letzten 3 Jahren Vorträge zum Thema für die Firmen Hevert, Hepart, SanOmega und Wörwag gehalten.

\section{Literatur}

[1] Gertsik L, Poland RE, Bresee C et al. Omega-3 fatty acid augmentation of citalopram treatment for patients with major depressive disorder. J Clin Psychopharmacol. 2012; 32(1): 61-64. doi: 10.1097/JCP. 0b013e31823f3b5f.

[2] Gröber U, Hollick, MF. Vitamin D: Die Heilkraft des Sonnenvitamins. Stuttgart: Wissenschaftliche Verlagsgesellschaft Stuttgart; 2014

[3] Grosso G, Galvano F, Marventano S et al. Omega-3 Fatty Acids and Depression: Scientific Evidence and Biological Mechanisms. Oxidative Medicine and Cellular Longevity. Volume 2014, Article ID 313570http://dx. doi.org/10.1155/2014/313570

[4] Jorde R, Sneve M, Figenschau Y et al. Effects of vitamin $D$ supplementation on symptoms of depression in overweight and obese subjects: randomized double blind trial. J Intern Med. 2008; 264(6):599-609. Epub 2008 Sep 10

[5] Krawczyk K, Rybakowski J. Augmentation of antidepressants with unsaturated fatty acids omega-3 in drug-resistant depression]. Psychiatr Pol. 2012; 46(4): 585-598

[6] May HT, Bair TL, Lappé DL et al. Association of vitamin $D$ levels with incident depression among a general cardiovascular population. Am Heart J. 2010; 159(6): 10371043

[7] Romieu I, Téllez-Rojo MM, Lazo M et al. Omega-3 fatty acid prevents heart rate variability reductions associated with particulate matter. Am J Respir Crit Care Med. 2005;172(12):1534-40. Epub 2005 Oct 6

[8] Schmiedel V. Natürlich Fisch! - Was Sie über Omega-3-Fettsäuren wirklich wissen müssen. Stuttgart: Trias; 2015

[9] Spitz J, Grant WB. Vitamin D - das Sonnenhormon. Murnau: Mankau; 2014

\section{ÜBER DEN AUTOR}

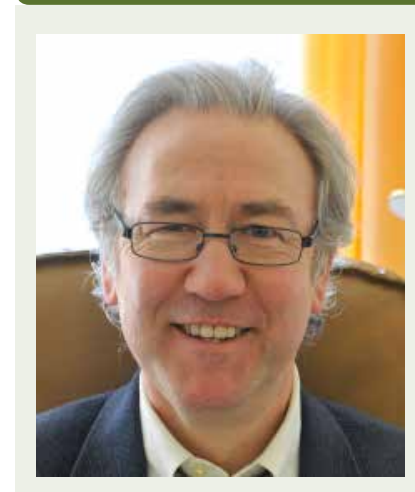

Volker Schmiedel war von 1996-2015 Chefarzt der Inneren Abteilung der Habichtswaldklinik (Kassel) und ist seit Oktober 2016 Arzt im ganzheitlichen Ambulatorium Paramed in Baar (Schweiz). Er ist zudem Fortbildungsleiter für „Naturheilverfahren“ der Medizinischen Woche, Mitherausgeber der Zeitschrift „Erfahrungsheilkunde“ und des „Leitfaden Naturheilkunde" sowie Autor zahlreicher weiterer naturheilkundlicher Bücher für Therapeuten und Laien.

KORRESPONDENZADRESSE

Dr. med. Volker Schmiedel

Facharzt für Physikalische und Rehabilitative

Medizin, Naturheilverfahren, Homöopathie

Paramed

Haldenstr. 1

CH-6340 Baar

E-Mail: v.schmiedel@paramed.ch 